\title{
Smart Resource Allocation Scheme for Mimo Microstrip Wireless Communication
}

Sandeepkumar Kulkarni kulkarni ( $\nabla$ sandeepbk_3897@rediffmail.com )

PDA College of Engineering: Poojya Doddappa Appa College of Engineering https://orcid.org/00000002-1521-4277

\section{Raju Yanamshetti}

PDA College of Engineering: Poojya Doddappa Appa College of Engineering

\section{Research Article}

Keywords: MIMO microstrip antenna, SNR, ANN, Data transmission

Posted Date: August 23rd, 2021

DOl: https://doi.org/10.21203/rs.3.rs-702646/v1

License: (1) This work is licensed under a Creative Commons Attribution 4.0 International License. Read Full License 


\title{
SMART RESOURCE ALLOCATION SCHEME FOR MIMO MICROSTRIP WIRELESS COMMUNICATION
}

\author{
Sandeepkumar kulkarni ${ }^{1} \quad$ Dr. Raju Yanamshetti
}

1.Department of E\&I, 2. Head of Department, Department of E\&CE,

HKE's Poojya Doddappa Appa College of Engineering, Kalaburagi-585102, Karnataka, INDIA

Email: sandeepbk 3897@ rediffmail.com,

Abstract: In this paper we have proposed a minimum noise shortest path determination scheme considering the amount of delay and energy consumed with respect to each path. An artificial neural network has been employed for classifying the minimum noise shortest path from the source to destination. A simulation work has been carried out with respect to different Signal-to-Noise (SNR) values in a thirty-node network with one Internet node and 100 bits of message length. Also, a comparison has been made between plain Dynamic Source Routing (DSR) and integrating the minimum noise shortest path algorithm with DSR. The simulation results show that with the increase of SNR, noise constraint in the path reduces, and data throughput increases.

Key words: MIMO microstrip antenna, SNR, ANN, Data transmission.

\section{INTRODUCTION}

The current wireless communication standard $4 \mathrm{G}$ faces various problems such as below par coverage, accommodating many channels, inferior quality, frequent dropping of connection and inflexibility. These challenges can be overcome by the arrival of next generation wireless communication standard, known as $5 \mathrm{G}$ which operates on millimeter wave band [I4][I5]. For the purpose of making $5 \mathrm{G}$ successful, various parameters regarding antenna design such as size of the antenna, bandwidth availability, antenna gain, power loss, traffic demand, and data rate must be improved [I6]. By varying the geometry and shape of Microstrip patch antenna, the above parameter values can be improved [I7]. Current research works on 5G wireless communication are extensively focusing MIMO Microstrip antennas. According to CISCO, there will be a ten-fold increase in traffic usage by smart phones and global requirement of data traffic will be multiplied by 7 times [I9]. By 2022, global IP traffic will reach an annual run rate of 4.8 zetta bytes per year [I8]. The following graph illustrates increase in the data traffic requirement from 2017 to 2022. 


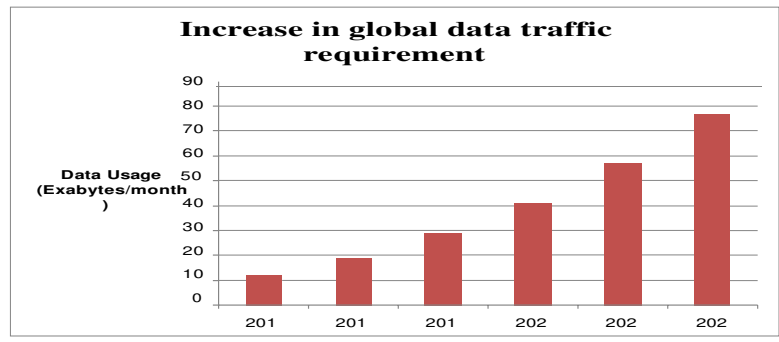

Fig. 1: Increase in global data traffic requirement from 2017 to 2022 [9]

But, traditional MIMO is not capable of meeting such high data usage supported by $5 \mathrm{G}$. That's why, a newer version of MIMO, known as massive MIMO has been considered for 5G technology. Where traditional MIMO uses 2 to 4 antennas, massive MIMO uses larger number of antennas. In addition to the characteristics of throughput and spectral efficiency increase of traditional MIMO, substantial increase in array gain is one of the fundamental characteristics of massive MIMO achieved using higher number of antennas [10].

\section{Problem of user scheduling}

In case of massive MIMO, concurrent transmission and receiving with large number of user devices can give rise to the problem of interference which consequently reduces the throughput. If by any chance, the number of user becomes more than the number of antennas, then the event of user scheduling can pose a threat to the system. So, a suitable user scheduling algorithm should be applied before precoding. A depiction of user scheduling has been given in figure 2 below.

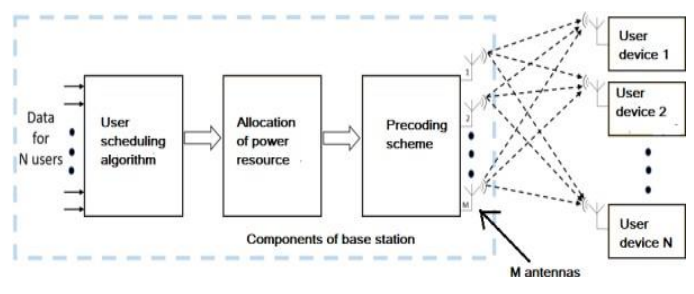

Figure 2: Application of user scheduling algorithm in massive MIMO [source: https://www.mdpi.com/1424-8220/20/10/2753/htm]

\section{Energy efficiency achievement}

Though the technique of massive MIMO leads to less amount of energy consumption along with the provision of gaining high amount of spectral efficiency, but there is always a possibility of high energy consumption due to use of a large number of antennas. There are 
various research works that are now focusing on minimizing energy consumption in MIMO by employing less complex and low cost algorithms for precoding, user scheduling, signal detection, channel approximation, and antenna as well as power amplifier design.

\section{Contributions in this research}

It is observed that use of deep learning-based algorithms can be beneficial for various massive MIMO operations for enhanced wireless communication. There are various existing and ongoing research works that are focusing on this area. A logical outcome is that if deep learning can enhance the performance of massive MIMO, then there are high possibilities of enhancing the performance of traditional MIMO wireless communication. Hence, this research work has carried out a simulation study to

- demonstrate how MIMO Microstrip antennas can be used in a network setting to get connected with the Internet and how such a system functions when many systems are accessing the Internet

- the Microstrip antenna that is being used here has been incorporated with soft computing, that is, the bandwidth and signal to noise (SNR) ratio are being adjusted depending upon the network parameters. Actually, various user devices are transmitting simultaneously and as a result there can be interference at the receiver end. Now, depending on the location of various sending devices, power, SNR and attenuation, a neural network-based classification algorithm is used to classify the received data in various suitable zones. So, the Microstrip antenna has been empowered with a simple decision-making soft computing system such that the antenna can switch to the most optimal configuration in order to maximize the throughput

- a comparison has been made between the functions of the Microstrip antennas when it is with and without soft computing.

\section{METHODOLOGY}

As MIMO -OFDM systems are extensively used in LTE network, here we are considering a heterogeneous LTE network for our experiment. 
In a heterogeneous network like LTE, communication via radio signal among user devices are basically managed by a base station along with the resource sharing and handover functions. $4 \mathrm{G}$ data carriers or WiMAX network is employed for connecting to the Internet. When there is scarcity of resources, the base station determines the optimal subcarrier with respect to noise and power constraints for all channels and provides to the node. Current resource requirements and resource constraints actually determine the optimal path in deviceto-device communication.

Objective of this work is to find out the shortest path for transmission which has minimum noise constraint. Transmission throughput will be increased if the least noise and high SNR link is selected for each data transmission.

\section{Block diagram}

The block diagram of the proposed system has been illustrated below.

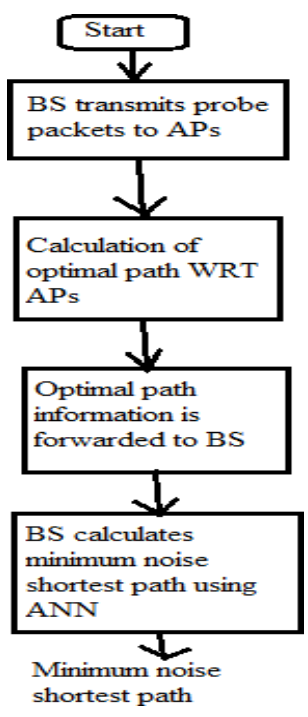

\section{Figure 4: Minimum noise shortest path calculation}

Before beginning of data transmission, the minimum noise shortest path has to be calculated. For this purpose, probe packets are transmitted by the base station to the access points with respect to variable data load and SNR. Various parameters such as packet loss, bit error rate, and latency are used to determine the optimal shortest path with respect to each access point. Then each access point forwards their findings to the base station. Using these outcomes, the base station utilizes ANN based minimum noise shortest path finding unit and determines the minimum noise shortest path at that instant. Depending on the noise interference, the ANN unit can suggest a new path or to retain the old path. 


\section{Algorithms for determining delay and energy consumed}

The ANN unit determines the minimum noise shortest path by taking two factors in consideration: amount of delay and consumption of energy with respect to the current path of data transmission.

Say, there are $\mathrm{N}$ nodes that are distributed over an area. The startup energy in each node is denoted as $E_{\mathrm{s}}$ and energy consumed for communication to one-hop neighbors is indicated as $E_{1 h}$. It should be mentioned that total energy consumption for communicating to adjacent neighbors should be less than startup energy which can be expressed as

$\sum E_{1 h}^{i} \kappa=E$, where $i=1,2, \ldots, N$

Algorithm for calculating amount of delay has been highlighted below.

- Let us consider total number of nodes as $\mathrm{N}=\left\{\mathrm{N}_{1}, \mathrm{~N}_{2}, \ldots, \mathrm{N}_{\mathrm{m}}\right\}$

- $E_{D}$ denotes energy consumption corresponding to packet delivery ration with respect to sending node. Again, consider that variable e denotes the energy of the path having minimum energy consumption.

That is $\mathrm{e}=\arg \min \left\{\mathrm{E}_{\mathrm{D}}\right\}$

- $\mathrm{D}$ is the minimum Euclidean distance between sending and receiving nodes.

$\mathrm{D}=\mathrm{e} \sum_{i=1}^{m}|N s-N r|$, where $N_{s}$ and $N_{r}$ denote sending and receiving nodes.

- Initially, the weight vector $W_{\text {old }}=D$

- The weights are updated according to the following equation $W_{\text {new }}=W_{\text {old }}+\mu\left(N_{s}-W_{\text {old }}\right)$, where $\mu$ denotes the learning rate, $0<=\mu<=1$

- By employing a linear function of distance, the resulting delay is measured. So, the path with least $E_{D}$ value is the required path

Next, algorithm for determining the energy consumption is detailed below.

- Say, there are $m$ available paths from sender to receiver.

- $\mathrm{C}$ is the counter of available paths. Initially $\mathrm{c}=1$.

- Let, E denotes minimum energy requirement per bit with respect to m number of paths.

Initially, $E$ is assigned maximum possible value 
$E=\sum_{i=1,2_{n} m} m i * E \max * L$, where $m_{i}$ denotes paths for $i=1,2 . . m, E_{\max }$ is maximum energy and $L$ denotes packet length in bits.

- Calculate $E_{\text {updated }}=\sum E_{i} * L$, where $E_{i}$ denotes energy consumption for one bit transmission

- $\quad$ Energy consumption of each path is updated as $E_{\text {updated }}=$ Euntil $\left|E_{\text {updated }}-E\right|<t$, where $t$ is a threshold value defined earlier

- Counter of paths is updated $c=c+1$

- Repeat four steps above

- All the paths are compared with respect to energy consumption and the least one is selected

The diagram below shows the ANN unit for determining minimum noise constraint path.

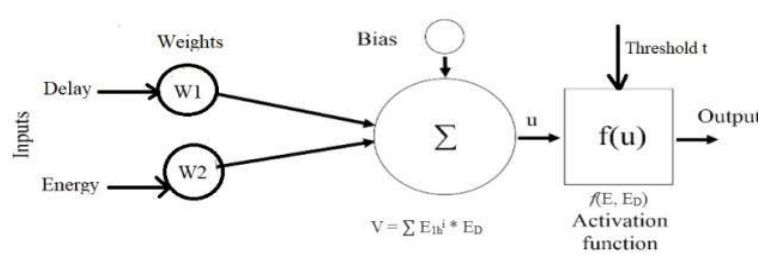

Figure 5: Artificial neural network structure source: https://bdtechtalks.com/2019/08/05/what-is-artificial-neural-network-ann/]

\section{RESULTS AND DISCUSSIOON}

\section{Simulation}

Simulation has been carried out using MATLAB. Various network topologies of 30 nodes have been generated. At different SNR value, delay and energy consumption have been examined and ANN based model has been used to calculate throughput.

Different parameters of the simulation and their values have been shown in the Table $-\mathrm{I}$ below.

\begin{tabular}{|c|c|}
\hline Parameter & Value \\
\hline Number of nodes & 30 \\
\hline Message length & 100 \\
\hline \multirow{4}{*}{ SNR } & -40 \\
\cline { 2 - 2 } & -20 \\
\cline { 2 - 2 } & -10 \\
\cline { 2 - 2 } & 10 \\
\cline { 2 - 2 } & 20 \\
\hline
\end{tabular}




\begin{tabular}{|c|c|}
\hline & 50 \\
\hline Internet node & 1 \\
\hline Rounds & 10 \\
\hline
\end{tabular}

Source: Author compilation

Simulation window at different parameter values

SNR -40 dB

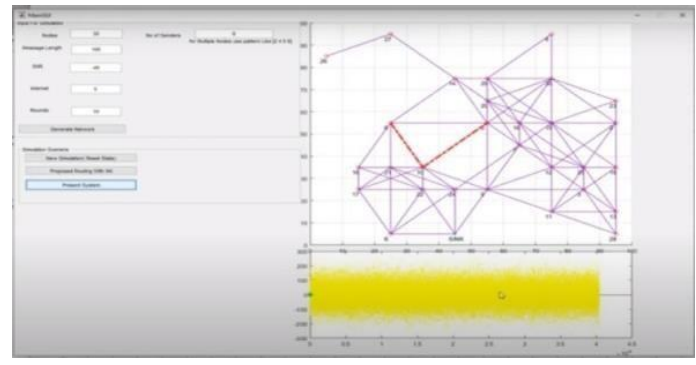

Figure 6: Simulation window at $-40 \mathrm{~dB}$

In the above diagram, total number of nodes is 30 . At this instant of time six nodes are actively communicating and SNR is $-40 \mathrm{~dB}$.

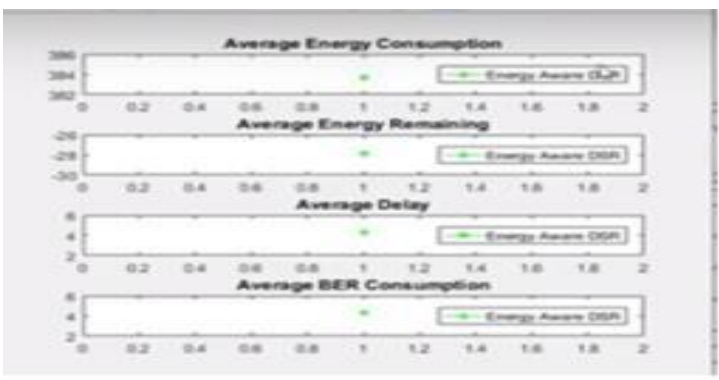

Figure 7: Delay and energy consumption status at $\mathbf{- 4 0} \mathrm{dB}$

From the above graph, it can be seen that at $-40 \mathrm{~dB}$, more energy consumption and more delay occur.

SNR -20dB

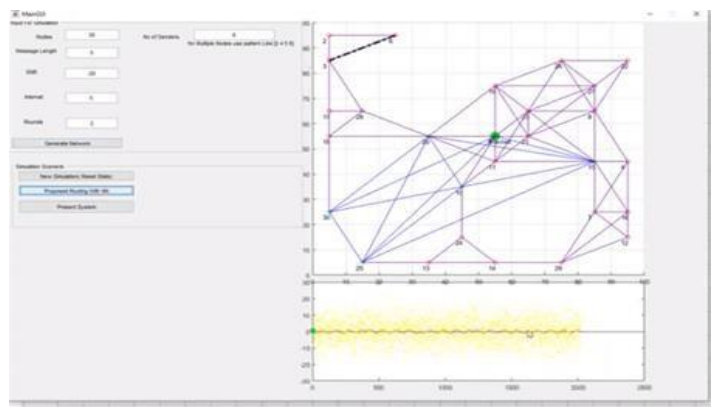

Figure 8: Simulation window at $-20 \mathrm{~dB}$ 
The simulation window above shows more noise and less signal strength at $-20 \mathrm{~dB}$. The graph below shows the status of delay and energy consumed at $-20 \mathrm{~dB}$.

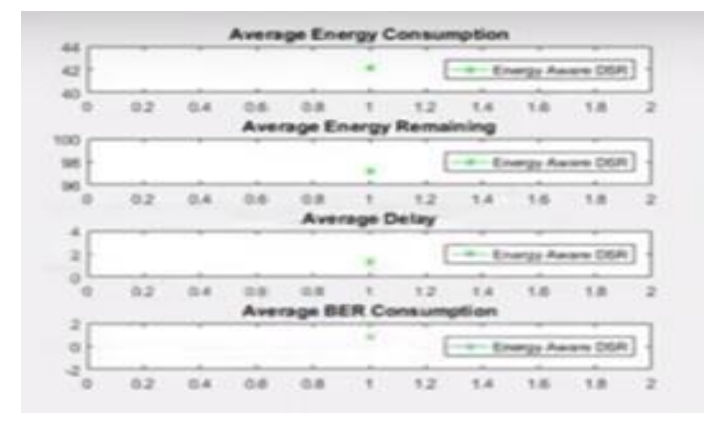

Figure 9: Delay and energy consumption status at $\mathbf{- 2 0} \mathrm{dB}$

\section{SNR 20 dB}

As shown in the figure below, amount of noise is very less and signal strength is quite high. Also, the signal propagation can be visualized clearly.

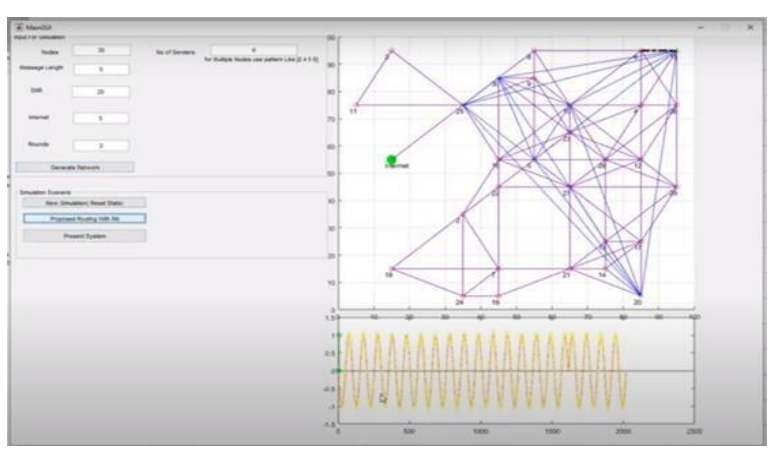

Figure 10: Simulation window at $20 \mathrm{~dB}$

The graph below demonstrates that average delay and energy consumption both are decreasing.

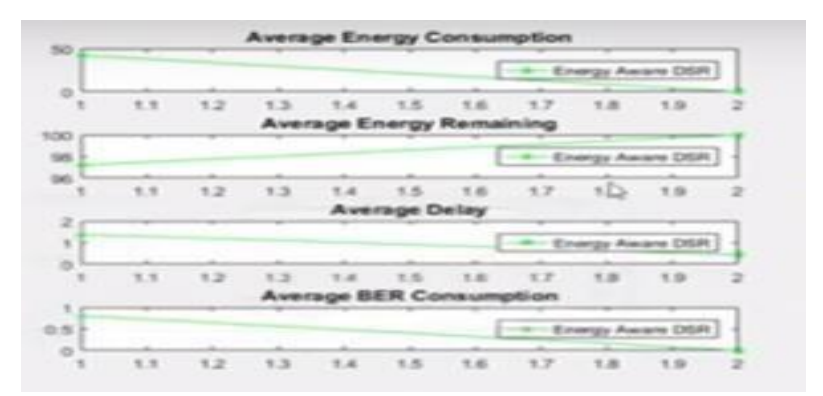

Figure 11: Delay and energy consumption status at $20 \mathrm{~dB}$ SNR 50 dB 


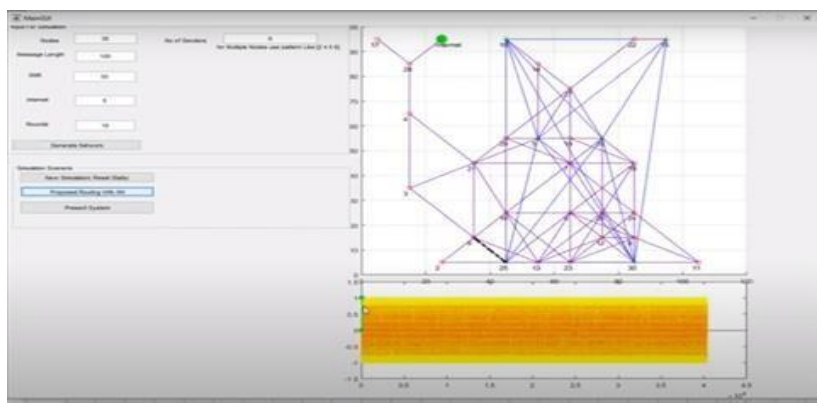

\section{Figure 12: Simulation window at $50 \mathrm{~dB}$}

The figure above shows the simulation window at SNR $50 \mathrm{~dB}$. From the graph below it can be observed that noise is very less and data transmission with maximum throughput is possible.

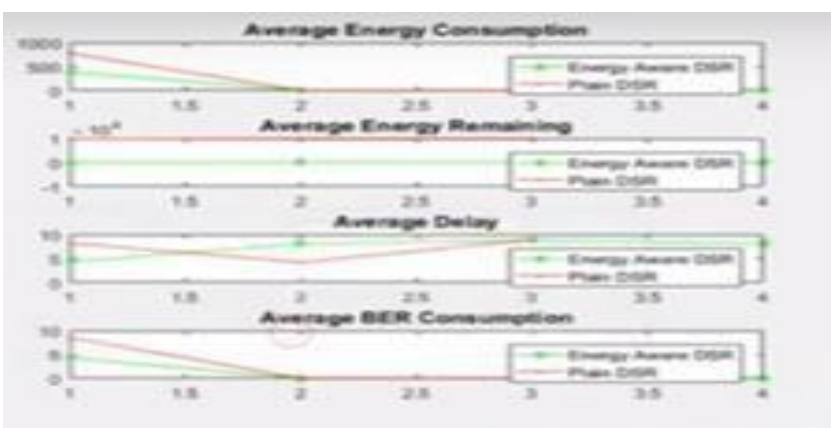

Figure 13: Delay and energy consumption status at $50 \mathrm{~dB}$

4. Bit error rate comparison for theoretical and simulated values

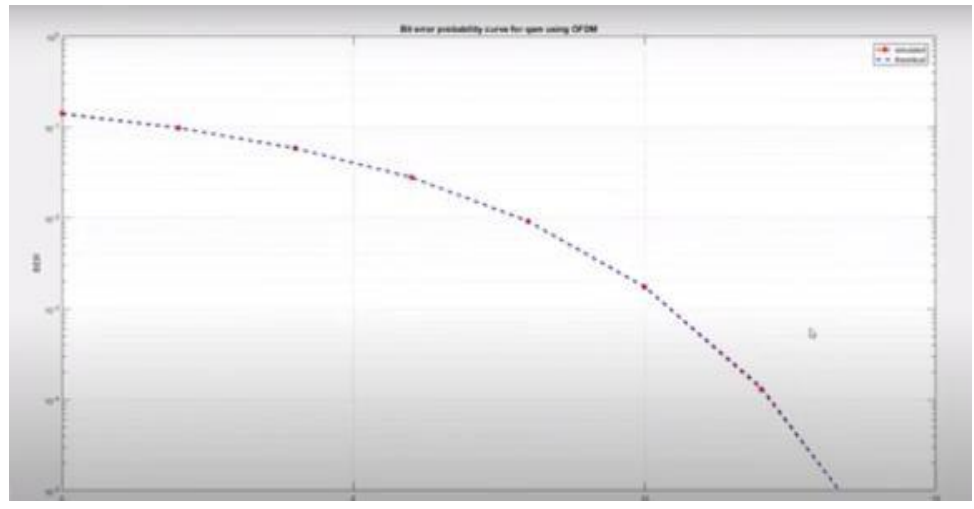

Figure 14: Bit error rate comparison with respect to theoretical and simulated values

Bit error rate compared for theoretical and simulated values considering QAM-OFDM system and fifty nodes into consideration. It can be observed from the above figure that simulated values are perfectly aligned with theoretical values proving that our simulation is working properly.

\section{CONCLUSION}


Increase in the number of user devices can pose a problem in optimal resource allocation strategies. Determining the minimum noise shortest path from the source to destination is a necessity for high data rate. So, in this paper has proposed a ANN based scheme for finding minimum noise constraint path. The neural network takes delay and energy consumption as input and decides for the noise-optimal path. The Base Station(BS) keeps a watch on the network performance and suggests the noise-optimal path according to current requirement. Simulation results show that the proposed scheme demonstrates better performance.

ACKNOWLEDGEMENT: I Sincerely thankful to my guide Dr. Raju Yanamshetti for bringing this article.

\section{AUTHOR'S PICTURE AND BIBLIOGRAPHY:}

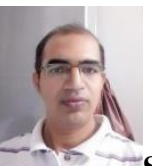

Sandeepkumar kulkarni received his Bachelor Degree in Electronics and Instrumentation from Gulbarga University, Gulbarga and Master Degree in Power Electronics from VTU -Belgaum in 2001 and 2006 respectively. In 2013, he joined in VTU-Belgaum as Ph.D part time student in MIMO microstrip antenna group and carrying his research work under the direction of Dr. Raju Yanamshetti, PDACE-Gulbarga. He has Membership of IEI, KRVP and KSP. Guided several UG and PG students and presented papers in National, International and UGC approved journals.

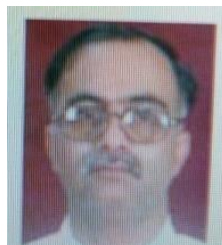

Dr. Raju Yanamshetti kulkarni received his Bachelor Degree in Electronics and Communication Engineering from Gulbarga University, Gulbarga and Master Degree in Power Electronics from Gulbarga University, Gulbarga and Ph.D in Electrical Engineering from Jadhavpur University in 1986, 1991 and 2012 respectively. He has Membership of ISTE, ISOI, and IETE. Presented several papers under National and International journals. Guiding several research scholars under various areas. Area of research in VLSI Design, real time embedded systems and solid state power controllers.

DECLARATION: 1. Funding information: No funding .

2. Conflict of interest: No such financial organizations have shown competing of interest in this work . 
3. Availability of data and material(data transparency): referred the papers

- Kalpesh Hiray, K. Vinoth Babu, "A Neural Network Based Channel Estimation Scheme for OFDM System', International Conference on Communication and Signal Processing, April 6-8, 2016, India, pp. 438-441

- Erik G. Larsson, Ove Edfors, Fredrik Tufvesson, Thomas L. Marzetta, "Massive MIMO for Next Generation Wireless Systems", IEEE Communications Magazine (Volume: 52, Issue: 2, February 2014), Page(s): 186 - 195, DOI: 10.1109/MCOM.2014.6736761

4. Code availability: Used mat lab 2016 version software.

5. Authors contributions: Author has worked on the smart reconfigurable antenna with 30 different nodes, selected best one which selected noise less operation and maximum data throughput.

Developing the Matlab software with relevant data for conducting test. Results of theoretical and practical are perfectly matched.

\section{REFERENCES}

[1] Gerard J. Foschini, "Layered Space-Time Architecture forWireless Communication in a FadingEnvironment When Using Multi-ElementAntennas", Bell Labs Technical Journal, Autumn 1996, pp. 41-59

[2] G.J. FOSCHINI and M.J. GANS, "On Limits of Wireless Communications in a Fading Environmentwhen Using Multiple Antennas", Wireless Personal Communications 6: 311335,1998

[3] A.R.G.Chandra Mouli.K, Dr. M.Satya Anuradha, Darshana Kuppili,Dattatreya Gopi, “Analysis of Rectangular and Triangular Microstrip Antenna Arrays using HFSS", 2015 13th International Conference on Electromagnetic Interference and Compatibility (INCEMIC), pp. $25-31$ 
[4] Pawan C. Mishra, Reena Sonkusare, "Performance Analysis of Simple Rectangular Microstrip Patch Antenna and Gap Coupled Rectangular Microstrip Patch Antenna”, 2017 International Conference on Intelligent Computing and Control (I2C2),

DOI: $10.1109 / \mathrm{I} 2 \mathrm{C} 2.2017 .8321847$

[5] Ezgi Kucuk, Burak Bayram, S. Taha Imeci, “E-Shaped Patch Antenna at 4.87 GHz”, 2018 International Applied Computational Electromagnetics Society Symposium (ACES), DOI: 10.23919/ROPACES.2018.8364313

[6] Zehai Wu, Biqun Wu, Zhenhua Su, Xiuyin Zhang, "Development challenges for 5G base station antennas", 2018 International Workshop on Antenna Technology (iWAT), DOI: 10.1109/IWAT.2018.8379163

[7] Srisuji. T, Nandagopal. C, “Analysis on Microstrip Patch Antennas for Wireless Communication", IEEE Sponsored 2nd International Conference On Electronics And Communication System (ICECS 2015), pp. 538-541

[8] S. Praveena, B.Murugeshwari, U.Surendar, R. Kayalvizhi, “A Review of Antenna Design for Millimeter Wave Range”, IOSR Journal of Electronics and Communication Engineering (IOSR-JECE) e-ISSN: 2278-2834, p-ISSN: 2278-8735, pp. 01-06

[9] Shing-Lung Steven Yang, Ahmed A. Kishk, and Kai-Fong Lee, "Frequency Reconfigurable U-Slot MicrostripPatch Antenna”, IEEE Antennas and Wireless Propagation Letters, Vol. 7, 2008, pp. 127-129

[10] Thomas Barnett, Jr., Shruti Jain, Usha Andra, Taru Khurana, "Cisco Visual Networking Index (VNI) Global and Americas/EMEAR Mobile Data Traffic Forecast, 2017-2022”,

Cisco Knowledge Network (CKN) Session, March, 2019

[11] Thomas Barnett, Shruti Jain, Usha Andra, Taru Khurana, "Cisco Visual Networking Index (VNI) Complete Forecast Update, 2017-2022, APJC Cisco Knowledge Network (CKN) Presentation, 2018

[12] Xiaoyong Wu, N.C. Beaulieu, Danpu Liu, “On Favorable Propagation in Massive MIMO Systems and Different Antenna Configurations”, IEEE Access (Volume: 5), Page(s): 5578 - 5593, DOI: 10.1109/ACCESS.2017.2695007

[13] Balasubramanian Gopalakrishnan and Nihar Jindal, "An Analysis of Pilot Contamination on Multi-UserMIMO Cellular Systems with Many Antennas”, 2011 IEEE 12th International Workshop on Signal Processing Advances in Wireless Communications, pp. 381-385 
[14] Erik G. Larsson, Ove Edfors, Fredrik Tufvesson, Thomas L. Marzetta, "Massive MIMO for Next Generation Wireless Systems”, IEEE Communications Magazine (Volume: 52, Issue: 2, February 2014), Page(s): 186 - 195, DOI: 10.1109/MCOM.2014.6736761

[15] Osvaldo Simeone, “A Very Brief Introduction to Machine LearningWith Applications to Communication Systems", IEEE Transactions On Cognitive Communications And Networking, Vol. 4, NO. 4, December 2018

[16] Jayden Booth, Sameeksha Katosh, Ahmed Alkhateeb, Ahmed Ewaisha, and Andreas Spanias, "Machine Learning for Reliable MIMO Systems”, IRES Summer Research, 2019 [17] Rongpeng Li, Zhifeng Zhao, Xuan Zhou, Guoru Ding, Yan Chen, ZhongYao Wang, and Honggang Zhang, “Intelligent 5G: When Cellular NetworksMeet Artificial Intelligence”, IEEE Wireless Communications (Volume: 24 , Issue: 5 , October 2017 ), Page(s): 175 - 183 [18] Athina Petropulu, Ruifeng Zhang, and Rui Lin, "Blind OFDM Channel Estimation ThroughSimple Linear Precoding”, IEEE Transactions On Wireless Communications, Vol. 3, No. 2, March 2004, pp. 647-655

[19] Juan I. Montojo, and Laurence B. Milstein, "Channel Estimation for Non-Ideal OFDM Systems", IEEE Transactions on Communications, Vol. 58, No. 1, January 2010, pp. 146-156 [20] Ming Liu, Matthieu Crussi`ere, and Jean-Franc,ois H'elard, "A Novel Data-Aided Channel Estimation withReduced Complexity for TDS-OFDM Systems”, IEEE Transactions on Broadcasting (Volume: 58, Issue: 2, June 2012), Page(s): 247 - 260

[21] Manasjyoti Bhuyan and Kandarpa Kumar Sarma, "MIMO-OFDM Channel Tracking using a DynamicANN Topology", World Academy of Science, Engineering and TechnologyInternational Journal of Electronics and Communication Engineering, Vol: 6, No: 11, 2012

[22] Cai Hua, Zhao Xiao-hui, "MIMO-OFDM channel estimation based on Neural Network", 2010 6th International Conference on Wireless Communications Networking and Mobile Computing (WiCOM), DOI: 10.1109/WICOM.2010.5600626

[23] Kalpesh Hiray, K. Vinoth Babu, “A Neural Network Based Channel Estimation Scheme for OFDM System', International Conference on Communication and Signal Processing, April 6-8, 2016, India, pp. 438-441

[24] Eren Balevi and Jeffrey G. Andrews, "Deep Learning-Based Channel Estimation forHigh-Dimensional Signals", arXiv: 1904.09346, 2019

[25] Raju Yanamshetti, Sandeepkumar Kulkarni "MEMS Based Circular Microstrip Patch Antenna at 2.4GHz for Wi-Fi Applications", ICEECCOT-2017, 
[26] Sandeepkumar Kulkarni Raju Yanamshetti “MIMO Reconfigurable Antennas for WiFi 2.4 GHz Communication",

IJITEE ,ISSN: 2278-3075, Volume-9 Issue-2S, December 2019

[27] WEI JIANG, AND HANS D. SCHOTTEN, "Neural Network-Based Fading ChannelPrediction: A Comprehensive Overview", Special Section on Artificial Intelligence for Physical-Layer WirelessCommunications, Volume 7, 2019, pp. 118112-118124 


\section{AUTHOR'S PICTURE AND BIBLIOGRAPHY:}

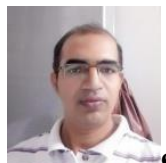

Sandeepkumar kulkarni received his Bachelor Degree in Electronics and Instrumentation from Gulbarga University, Gulbarga and Master Degree in Power Electronics from VTU -Belgaum in 2001 and 2006 respectively. In 2013, he joined in VTU-Belgaum as Ph.D part time student in MIMO microstrip antenna group and carrying his research work under the direction of Dr. Raju Yanamshetti, PDACE-Gulbarga. He has Membership of IEI, KRVP and KSP. Guided several UG and PG students and presented papers in National, International and UGC approved journals.

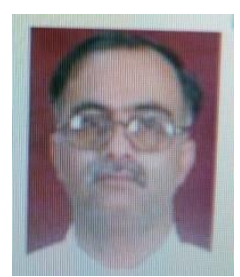

Dr. Raju Yanamshetti kulkarni received his Bachelor Degree in Electronics and Communication Engineering from Gulbarga University, Gulbarga and Master Degree in Power Electronics from Gulbarga University, Gulbarga and Ph.D in Electrical Engineering from Jadhavpur University in 1986, 1991 and 2012 respectively. He has Membership of ISTE, ISOI, and IETE. Presented several papers under National and International journals. Guiding several research scholars under various areas. Area of research in VLSI Design, real time embedded systems and solid state power controllers. 